\section{CREATING CULTURES OF SAFETY: PARADOXICAL EFFECTS OF IDENTICAL SAFETY CULTURE INTERVENTIONS ON INPATIENT HOSPITAL UNITS}

Howard Chiou. Emory University, United States

10.1136/bmjqs-2015-IHlabstracts.28

Background Our knowledge of how to create safety cultures is extremely limited. This research utilized an unusual opportunity to longitudinally study how the same intervention-an evidence-based bundle, including bedside multidisciplinary rounds and a quality-safety checklist-altered culture differently on three inpatient hospital units.

Objectives Using methods from both anthropology and quality improvement, this research evaluates the dynamic process of implementing a highly complex culture change intervention by: (1) describing the units at baseline, (2) characterizing the transformation of units during implementation, and (3) identifying the activities and contextual factors that accelerated or hindered the implementation process.

Methods Three inpatient internal medicine units in the US and Australia were studied over two years. Data collection included over 420 hours of ethnographic participant observation, 102 semi-structured interviews with hospital staff, and 141 safety culture questionnaires. Qualitative data was coded and analyzed using modified grounded theory, and triangulated against the quantitative using iterative constant comparison and cross-case analysis.

Results The intervention successfully disrupted cultural norms on one unit, but unexpectedly failed to do so at another. The implementation was well-resourced and consistent between units. However, the failed unit suffered from an inadvertent reinforcement of hierarchy within rounds, mutations in the meaning of checklists, and interactions with local hierarchies and conceptions of nurse-patient relationships.

Conclusions This research informs the design of subsequent culture change interventions, as reports of failure to change culture are rare in the literature. This study uniquely expands previous understanding by building a framework based in the social sciences for understanding and changing safety culture. 